Case Report

\title{
Evaluation of Fever of Unknown Origin under the COVID-19 Pandemic Condition
}

\author{
Melis ARDIÇ ${ }^{1}$, Mert TÜRK ${ }^{1}$, Nizameddin $\mathrm{KOCA}^{1}$
}

${ }^{1}$ Health Sciences University, Bursa Yüksek İhtisas Training and Research Hospital, Department of Internal Medicine, Bursa, Turkey

\begin{abstract}
HIV infection can manifest itself in many different clinical situations. In a pandemic such as COVID, when fever is of diagnostic importance and manifests itself with different clinical pictures, it is very difficult to distinguish the true cause of high fever. In this case report, we shared the process of diagnosing HIV infection in a patient with type 1 diabetes who presented with fever and neurological symptoms under pandemic conditions.
\end{abstract}

Turk J Int Med 2021;3(Supplement 1):S82-S84

DOI: $\underline{10.46310 / \text { tjim. } 877015}$

Keywords: HIV, COVID-19, fever of unknown origin, diabetic ketoacidosis

\section{Introduction}

The increased diagnosis and treatment methods developed in the last 30 years improved the detection of human immunodeficiency virus (HIV) infected individuals at an earlier stage which increased the life expectancy and decreased the transmission rate of these patients. ${ }^{1,2}$ Although patients diagnosed with HIV mostly present with infectious mononucleosis-like symptoms, some patients may present with unusual clinical presentations. 3 In this case, we aimed to present a 23-year-old diabetic patient (type 1 diabetes mellitus: T1DM) admitted to hospital with generalized tonic clonic seizure and fever of unknown origin who finally diagnose with HIV infection and recovered after receiving antiretroviral therapy.

\section{Case Report}

A 23-year-old male, who had been followed up with intensive insulin therapy for 12 years with the diagnosis of T1DM, admitted to the emergency department with complaints of high fever, nausea-vomiting and headache. Under the

Received:February 8,2021;Accepted:March 6,2021;Published Online:March 6, 2021

\section{Address for Correspondence:}

Melis ARDIC 


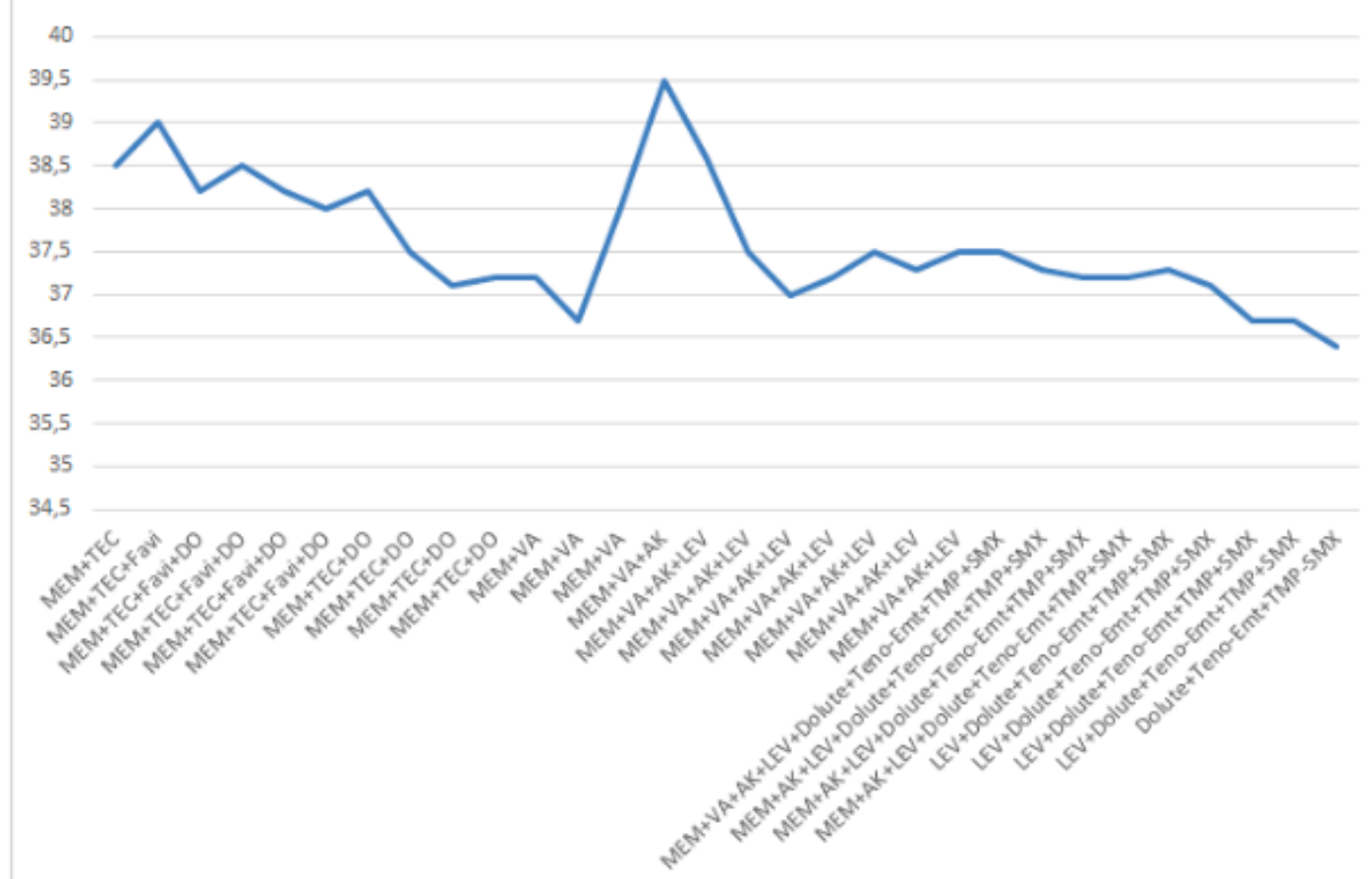

Figure 1. Fever response to antibiotics and antiviral treatments. MEM: Meropenem, TEC: Teicoplanine, Favi: Favipiravir, DO: Doxycycline, VA: Vancomisine, AK: Amikacin, LEV: Levofloxacine, Dolute: Dolutegravir, Teno-Emt: Tenofovir disoproxil + emtricitabine, TMP-SMX: Trimethoprime sulphamethaxasole

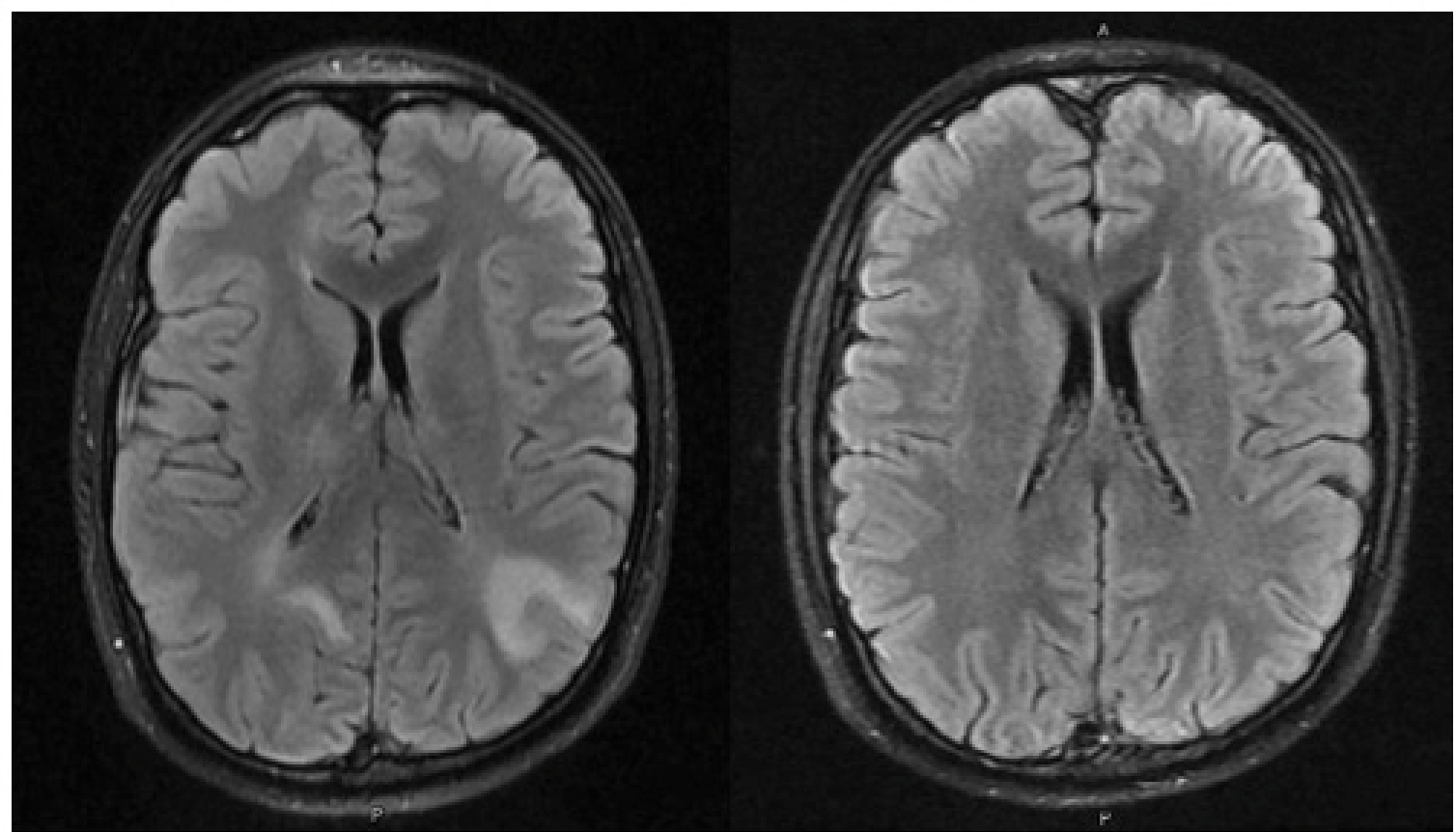

Figure 2. The comparison of the cranial MRI of the patient before (left) and after (right) antiretroviral treatment

pandemic condition, the patient was evaluated for COVID-19 and admitted to the intensive care unit after the observation of a generalized tonic clonic seizure in the emergency room. Although, he had his first generalized tonic clonic seizure six months ago he did not admit neurologist for accurate diagnosis and treatment. On physical examination, he had a fever $\left(38.6^{\circ} \mathrm{C}\right)$, tachycardia (pulse 110 beats/min). Chest auscultation showed decreased respiratory sounds at lower zones. Neurological examination revealed retrograde amnesia and a positive Babinski reflex on the left.

In laboratory examinations, plasma glucose level was $420 \mathrm{mg} / \mathrm{dL}$, +3 ketone in the urine, 
arterial pH: 7.18, ferritin >2,000, CRP: $109 \mathrm{mg} /$ $\mathrm{dL}$, and procalcitonin $>50 \mathrm{pg} / \mathrm{mL}$. Minimal pleural effusion in both hemithoraxes, and a light ground glass density with unclear borders in the right lung middle lobe lateral segment posterior peripheral area was observed in chest computed tomography (CT). In cranial magnetic resonance imaging (MRI), high signal areas and mild edema signals observed in the ventrals of both temporal lobes and temporoparietal lobes, subcortical areas in $\mathrm{T} 2$ and Flair were noted, and it was reported that those findings may be associated with metabolic events or encephalomyelitis.

Diabetic ketoacidosis treatment protocol was initiated. Meropenem and teicoplanin antibiotherapy was started empirically due to increased acute phase reactants including procalcitonin and fever. Favipiravir was started with the pre-diagnosis of COVID-19 pneumonia due to the ground-glass densities in chest CT. The patient, whose fever did not respond to treatment during the follow-up, was evaluated as pericarditis, and colchicine and ibuprofen treatment was initiated. The treatments and fever response of the patient are shown in Figure 1. Since the patient's headache complaint did not resolve and retrograde amnesia was observed in the repeated mini mental test the treatment was changed with the pre-diagnosis of encephalitis. The anti-HIV test requested from the patient, whose fever and neurological findings continued despite the treatment changes, was reported as positive. HIV-RNA was detected as 115,200 copies/mL, and the CD4/CD8 ratio of the patient was measured as 0.23. Lumbar puncture was performed on the patient to evaluate in terms of opportunistic infections. No cells were observed in the cell count of the sent cerebrospinal fluid (CSF) samples, glucose, protein and LDH levels were observed as $113 \mathrm{mg} / \mathrm{dL}$ (simultaneous blood glucose $139 \mathrm{mg} / \mathrm{dL}$ ), $50 \mathrm{mg} / \mathrm{dL}$, and $22 \mathrm{U} / \mathrm{L}$, respectively. Bacteriological, mycological and mycobacteriological growth was not detected in CSF cultures. ARB and VDRL tests were negative in CSF, meningitis-encephalitis panel (CMVDNA, VZV-DNA, HSV1-DNA, HSV2-DNA, HHV6-DNA, Enterovirus-RNA, H. influenzae,
N. meningitidis, S. pneumoniae, C. neoformans) resulted as negative. Rose-Bengal test performed for brucellosis was also negative. After the initiation of trimethoprim-sulfamethoxazole and dolutegravir, tenofovir disoproxil+emtricitabine for opportunistic infection prophylaxis and antiretroviral therapy, the patient's fever decreased. No pathology was observed in the control cranial MRI taken 1 week after the patient's neurological examination showed improvement. The comparison of the cranial MRI of the patient before and after antiretroviral treatment is given in Figure 2.

\section{Discussion}

Despite the multiple antibiotic treatments given for high fever, as seen in Figure 1, it was observed that the high fever and neurological findings of the patient continued. Patient's fever decrease just after the initiation of anti-retroviral treatment. Although there is no response to broad spectrum antibiotic treatments given for the fever symptom, which is often associated with opportunistic infections during the course of AIDS, it can be speculated that HIV itself may cause high fever, based on the regression of fever just after the initiation of antiretroviral treatment.

\section{Conflict of Interests}

Authors declare that there are none.

\section{Acknowledgment}

This study has been presented in $17^{\text {th }}$ Uludag Internal Medicine National Winter Congress, $6^{\text {th }}$ Bursa Family Medicine Association National Congress, $11^{\text {th }}$ Uludag Internal Medicine Nursing Congress, 5-7 March 2021, Bursa, Turkey.

\section{References}

1. Alexander TS. Human immunodeficiency virus diagnostic testing: 30 years of evolution. Clin Vaccine Immunol. 2016 Apr 4;23(4):249-53. doi: 10.1128/CVI.00053-16.

2. Mayer KH, Venkatesh KK. Antiretroviral therapy as HIV prevention: status and prospects. Am J Public Health. 2010 Oct;100(10):1867-76. doi: 10.2105/AJPH.2009.184796.

3. Moylett EH, Shearer WT. HIV: clinical manifestations. J Allergy Clin Immunol. 2002 Jul;110(1):3-16. doi: 10.1067/ mai.2002.125978. 